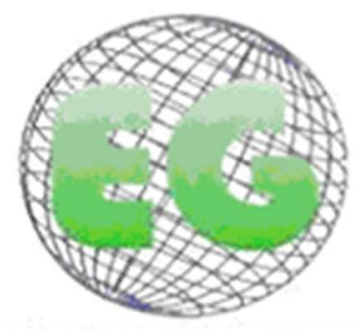

ISSN 1695-6141 $\mathbf{N}^{\circ} 63$

\title{
ORIGINALES
}

\section{Relación entre sesiones de atención plena y el nivel de estrés en estudiantes universitarios}

Relationship between full attention sessions and the stress level in university students

\section{Karla Villarroel-Carrasco ${ }^{1}$}

Consuelo Cruz-Riveros ${ }^{2}$

\author{
${ }^{1}$ Magíster, Enfermera de IAAS en Hospital San José. Santiago, Chile. kvillarroelk@gmail.com \\ 2 Magíster, Docente Facultad de Salud, Carrera de Enfermería, Universidad Santo Tomás, sede \\ Antofagasta, Chile.
}

\section{https://doi.org/10.6018/eglobal.442391}

Recibido: $4 / 09 / 2020$

Aceptado: $3 / 03 / 2021$

\section{RESUMEN:}

Introducción: El estrés académico constituye una problemática importante de abordar en la actualidad, la población estudiantil durante los últimos 10 años se ha enfrentado a diversos estresores desencadenando signos y síntomas, que se exacerban al enfrentarse a ciertas asignaturas. Estas asignaturas son las que incluyen metodologías nuevas en pro de lograr los resultados de aprendizajes esperados, dentro de las cuales encontramos la simulación clínica, la cual busca situaciones cercanas a lo real.

Objetivo: Determinar la relación existente entre el número de sesiones Mindfulness sobre el nivel de estrés frente a la metodología de simulación clínica en estudiantes universitarios de 3ero y 4 to nivel de la Carrera de Enfermería en una Universidad privada de la ciudad de Antofagasta, norte de Chile.

Método: Cuantitativa, experimental de tipo cuasi experimental con medición de test post-intervención. Muestra de 58 estudiantes. Para procesar la información se utilizaron pruebas de normalidad a las variables de la escala aplicada (SISCO) mediante Shapiro-Wilks, ANOVA, prueba de Tukey y pruebas $\mathrm{T}$ (hombres y mujeres).

Resultados: El nivel de estrés presentado en la muestra fue destribuido de la siguiente manera: $5 \%$ leve, $79 \%$ moderado, $16 \%$ profundo. Existiendo una asociación entre estrés y género, así como diferencias de medias en reacciones físicas y comportamentales en estudiantes que asisten a 4 sesiones (reacciones físicas: 0.91 y reacciones compartimentales: 1.07).

Conclusión: A mayor número de sesiones de Mindfulness, disminuye el nivel de estrés en las áreas de reacciones físicas y comportamentales.

Palabras claves: Estudiantes, atención plena, estrés, simulación.

\section{ABSTRACT:}

Introduction: Academic stress constitutes an important problem to be addressed today, the student population during the last 10 years has faced various stressors triggering signs and symptoms, which are exacerbated when facing certain subjects. These subjects are those that include new methodologies in order to achieve the expected learning results, within which we find clinical simulation, which looks for situations close to the real thing. 
Objective: To determine the relationship between the number of Mindfulness sessions on the level of stress compared to clinical simulation methodology in university students of 3rd and 4th level of Nursing Career at a private University Antofagasta, Chile.

Method: Quantitative, quasi-experimental experimental with post-intervention test measurement. A sample of 58 students was studied. To process the information, normality tests were applied to the variables of the applied scale (SISCO) using Shapiro-Wilks, ANOVA, Tukey's test and T tests (men and women).

Results: The stress level presented in the sample is distributed as follows: $5 \%$ mild, $79 \%$ moderate, $16 \%$ profound. There is an association between stress and gender, as well as mean differences in physical and behavioral reactions in students who attend 4 sessions (physical reactions: 0.91 and compartment reactions: 1.07).

Conclusion: It is shown that the greater the number of Mindfulness sessions, the lower the stress level in the areas of physical and behavioral reactions.

Key words: Students, mindfulness, stress, simulation

\section{INTRODUCCIÓN}

Actualmente, una de las problemáticas más experimentadas en la juventud chilena y que está afectando alarmantemente a la población estudiantil es el denominado estrés académico. Los autores Jerez y Oyarzo, señalan respecto a esta definición lo siguiente: "[..] desde los grados preescolares hasta la educación universitaria de postgrado, y son desencadenados durante el período de aprendizaje" (1). En Latinoamérica, según Román, la incidencia del estrés académico, en los estudiantes universitarios, está presente en un $67 \%$ (2). El año 2015 , fue realizada una investigación, en una Universidad del Sur de Chile, dirigida a estudiantes de distintas carreras del área de la salud (kinesiología, nutrición y dietética, fonoaudiología y enfermería, 314 personas participaron del estudio). El estudio evidenció que el $98 \%$ de los participantes están afectados por el estrés académico ${ }^{(1)}$.

Si consideramos lo datos mencionados anteriormente, es posible afirmar que en algún momento esta población estudiantil del área de la salud ingresará al mundo laboral. Al respecto Mejías y Manriquez, indican que: "[..] uno de los colectivos más expuestos al estrés, producto de las características de su trabajo son los profesionales de la salud, en especial el profesional de enfermería" (3). No obstante, la sobrecarga física y emocional es iniciada en la etapa de pregrado, según señala Duarte ${ }^{(4-6)}$.

Las evidencias anteriores invitan a reflexionar sobre el estrés en los estudiantes de las carreras del área de la salud y cómo está distribuida la carga académica, considerando que existen asignaturas de cátedras y laboratorios. Además, en los últimos años se integró como parte de las asignaturas el proyecto de simulación clínica, evaluada de forma positiva, por el fortalecimiento de los conocimientos de estudiantes mediante la metodología del ensayo - error, permitiendo a los estudiantes "[..] la oportunidad de realizar una práctica análoga a la que desarrollarán en la realidad asistencial" ${ }^{(7)}$.

No obstante, esta metodología también presenta desventajas. Una de ellas es la necesidad de un trabajo previo con un guión preestablecido antes del escenario de simulación (deben ser casos reales), sin embargo, no reflejan cabalmente lo que ocurre en la realidad.

Respecto a las investigaciones desarrolladas a nivel mundial a la población de pregrado en el área de salud, algunos estudios han utilizado el desarrollo de 
herramientas personales para enfrentar el estrés, destacando la realizada el año 2015, por Song y Lindquist, citado por Sansó et al. ${ }^{(8)}$ en el sur de Corea. Esta investigación consideró una muestra de 50 estudiantes de Enfermería, demostrando que el programa MBSR (conciencia plena) presenta como resultados "[...] efectos saludables sobre la depresión, la ansiedad, el estrés y la atención". Por otra parte, el año 2011, en Tasmania, también fue desarrollada una investigación a estudiantes de pre grado del área de salud, con una muestra de 66 estudiantes de medicina, con un grupo control y otro experimental, fueron aplicadas intervenciones con meditación guiada, logrando una disminución significativa en el estrés ${ }^{(9)}$. Las mismas variables son replicadas en el estudio realizado por la Universidad Autónoma de México en el año 2017, entregando un resultado positivo al empleo de Mindfulness en el nivel de estrés académico ${ }^{(10)}$.

En efecto, Mindfulness permite para quienes lo practican, contemplar sentimientos y pensamientos, haciéndolos conscientes, rompiendo así, la triada pensar, sentir y actuar, permitiendo el logro de la autogestión de dichas señales, facilitando que la persona pueda elegir de manera eficaz su forma de actuar frente a diversas situaciones, evitando la influencia negativa del estrés. Como indica Creswell, citado por Moreno (11) "[..] los efectos de la práctica del "estar atento" sobre el sistema nervioso se relacionan con la promoción de un estado de relajación del sistema nervioso periférico y la activación del sistema nervioso central". También, existen reportes, donde el entrenamiento en atención plena mejora la conectividad cerebral $^{(12)}$. Lo anterior, provocaría un incremento en la materia gris de las áreas del cerebro involucradas en los procesos de aprendizaje, memoria y regulación de las emociones $^{(13)}$.

Si bien Mindfulnes es un método que presenta resultados positivos, presenta la dificultad de poca literatura crítica en relación a la medición de estrés en simulación clínica y técnicas para enfrentar las situaciones desencadenantes. Por lo tanto, el objetivo de la investigación es determinar la relación existente entre el número de sesiones de Mindfulness sobre el nivel de estrés frente a la metodología de simulación clínica en estudiantes universitarios de 3 ero y 4 to nivel de la carrera de Enfermería en una Universidad privada de Antofagasta, norte de Chile.

\section{MÉTODOS}

El estudio es cuasi experimental de diseño estático con dos grupos con medición post-intervención. La selección de la muestra es obtenida durante el primer semestre del año 2019, por medio de la inscripción de los estudiantes que cursaron la asignatura "Metodología de simulación clínica", por lo tanto, esta etapa no es aleatoria. El tamaño muestral fue de 58 personas en total, permitiendo realizar la intervención con un nivel de confianza del $95 \%$ y un nivel de significación del $5 \%$.

La muestra, fue dividida en dos grupos de 29 estudiantes (control e intervención). La conformación de los grupos fue de manera natural, dada por la composición de los grupos de simulación clínica (figura 1).

Los criterios de inclusión fueron tener más de 18 años, cursar tercer o cuarto nivel de la carrera de enfermería, estar inscritos en asignaturas de metodología de simulación 
clínica, y acceder a firmar voluntariamente el consentimiento informado (los estudiantes debían completar el $70 \%$ y más del cuestionario).

Figura 1. Diagrama de flujo de selección de los participantes

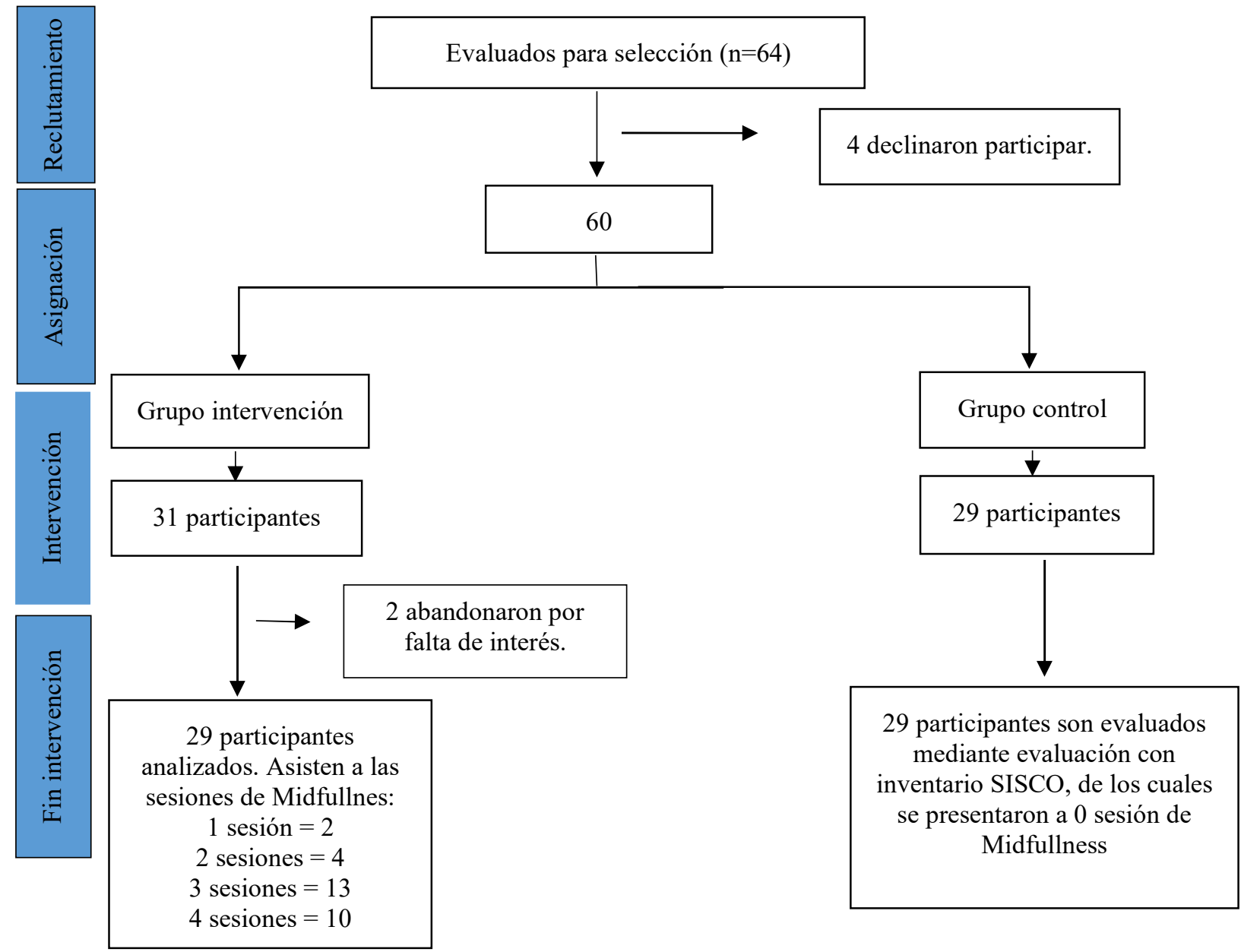

Fuente: Elaboración propia a partir de los datos

\section{Nivel de estrés}

Es evaluado mediante el inventario SISCO. Esta medición está configurada por 31 ítems, distribuidos en los siguientes componentes (12): Situaciones estresantes, reacciones físicas, reacciones psicológicas, reacciones compartimentales y estrategias de afrontamiento. La confiabilidad del instrumento fue medida mediante 2 pruebas estadísticas: confiabilidad por mitades, entregando un resultado de 0,87 y una confiabilidad en alfa de Cronbach de 0,90.

\section{Sociobiodemográficas}

Las variables consideradas fueron las siguientes: sexo (masculino/femenino), situación laboral (trabaja si/no), hijos (si/no) y estilo de vida (horas de sueño, horas de ocio, horas de ejercicio físico, y consumo de tabaco (si/no)). 


\section{Consideraciones éticas}

El proyecto fue autorizado por el comité de Ética Científico Macrozona Norte, Universidad Santo Tomás, sede lquique y por la Directora de la Carrera de Enfermería. El resguardo de los individuos es sustentado en los siete requisitos éticos del Sr. Ezekiel Emanuel (valor social, validez científica, selección equitativa del sujeto, proporción favorable de riesgo-beneficio, evaluación independiente, consentimiento informado y respeto a los sujetos inscritos). Además, se adhiere al cumplimiento de la legislación vigente.

\section{Intervenciones}

A continuación, se detallan las sesiones realizadas con los objetivos de cada una de ellas:

a. Sesión 0: En el primer encuentro, es realizada la introducción al Mindfulness con la aplicación de estas técnicas en educación y salud, revisando la metodología de trabajo para las sesiones posteriores.

b. Sesión 1: El objetivo del encuentro es conocer y comprender el concepto de resiliencia para afrontar el estrés académico. Efectuada la introducción, es expuesto un Power Point (PPT) en relación a la temática de la resiliencia, realizándose lectura en voz alta de dos frases. Luego, los estudiantes reflexionaron en grupo y elaboraron una conclusión del tema planteado. Posteriormente, desarrollan un diálogo grupal y conclusión del tema aclarando la terminología y el aporte en el manejo del estrés académico. Para finalizar, los estudiantes son invitados a realizar una práctica diaria de al menos cinco minutos de respiración consciente en la temática de resiliencia (entrega de la hoja de registro para vaciar la experiencia personal de la semana y acordar fecha para la próxima sesión).

c. Sesión 2: Al inicio del encuentro compartieron las experiencias de la semana y desarrollan la respiración consciente (el estudiante sentado en su silla, con los ojos cerrados). El objetivo del encuentro es la identificación y reconocimiento de sus emociones. Luego, con los estudiantes plenamente conscientes, exposición en PPT de dos frases referentes a la temática de las emociones. Lectura del texto en voz alta y discusión grupal donde son identificadas las emociones, elaborando una conclusión del tema (20 minutos). Posteriormente, exposición de los estudiantes, generando un diálogo grupal y conclusión del tema, reconociendo la importancia de lograr identificar las emociones, hacerlas conscientes, discriminando las positivas y negativas, para así expresarlas en conciencia plena. Para finalizar, invitación a los estudiantes de realizar una práctica diaria de al menos cinco minutos de respiración consciente conectando las emociones del momento (entrega de la hoja de registro para vaciar la experiencia personal de la semana y acordar fecha para la próxima sesión).

d. Sesión 3: Al inicio del encuentro compartieron las experiencias de la semana y desarrollan la respiración consciente (el estudiante sentado en su silla, con los ojos cerrados). El objetivo del encuentro es la identificación y reconocimiento del estrés en situaciones donde lo han experimentado, identificando las sensaciones corporales en el momento estresante. Luego, con los estudiantes plenamente conscientes, exposición en PPT de tres frases en referencia a la temática de las emociones. Lectura del texto en voz alta, discusión grupal, identificando el estrés en su vida 
académica (20 minutos). Exposición de los estudiantes, reconociendo la existencia del estrés académico, cómo se va desarrollando y las respuestas frente al tema. Para finalizar, invitación a los estudiantes a realizar una práctica diaria de al menos cinco minutos de respiración consciente conectando las situaciones estresantes en su vida estudiantil (entrega de la hoja de registro para vaciar la experiencia personal de la semana y acordar fecha para la próxima sesión.

e. Sesión 4: al inicio del encuentro comparten las experiencias de la semana y desarrollan la respiración consciente (el estudiante sentado en su silla, con los ojos cerrados). El objetivo del encuentro es la identificación y reconocimiento del tiempo como un preciado instrumento. Luego, con los estudiantes plenamente conscientes, exposición en PPT de dos frases en referencia al beneficio de la carga académica. Lectura del texto en voz alta, discusión grupal, proponiendo la forma de gestionar el tiempo en la vida académica para beneficio de los niveles de estrés (20 minutos). Exposición de los estudiantes, reconociendo la importancia de lograr una mejor distribución del tiempo en beneficio de las actividades académicas y como medio para enfrentar el estrés. Para finalizar, invitación a los estudiantes a continuar con la práctica de consciencia plena para beneficio personal y también como una forma de perpetuar las sensaciones positivas que han descubierto con ayuda de las sesiones (Aplicación del intrumento escala de nivel de estrés de forma anónima, en un tiempo de 10 minutos).

\section{Análisis estadístico}

Para el análisis estadístico fue construida una matriz de datos con el programa Microsoft Excel 2010, exportándose al paquete estadístico IBM SPSS v21, con el objetivo de obtener un análisis descriptivo y estadístico multivariados. Con lo anterior, son construidas las tablas de frecuencias para describir las variables estudiadas.

Para el procesamiento de datos, fueron utilizadas las pruebas de normalidad para escala SISCO SHAPIRO - WILKS. Para identificar la prueba a realizar análisis paramétricos o no paramétricos. ANOVA, prueba de homogeneidad de varianzas. TUKEY, para comparar pares de grupos específicos. Pruebas T, con un nivel de significación Alfa ( $\alpha$ ) de $5 \%$, el nivel de confianza es del $95 \%$.

\section{RESULTADOS}

\section{Características de la muestra}

Las características biosociodemográficas de la muestra general evidencian por sexo que el $81 \%$ son mujeres. En relación a la situación laboral de los estudiantes, el $76 \%$ declara no trabajar. La variable presencia de hijos, 4 personas responden positivamente $(6 \%)$. En los estilos de vida, los valores según grupo son: las horas de sueño presentan una media de $6 \mathrm{hrs}$, horas de ocio una media de 2,8 hrs y ejercicio una media de 2 hrs. En una muestra de 58 estudiantes (tabla 1).

Respecto a la evaluación del nivel de estrés post- intervención, la mayor cantidad de estudiantes presenta un nivel de estrés moderado. Destacando en el grupo control (0 sesión) 8 estudiantes con un nivel de estrés profundo (tabla 1). 
Según la frecuencia de asistencia a los talleres de Midfullnes, la muestra se comporta de la siguiente manera: 0 sesión: 29 estudiantes, sesión 1: 2 estudiantes, sesión 2: 4 estudiantes, sesión 3: 13 estudiantes y sesión 4: 10 estudiantes (tabla 1).

Tabla 1. Descripción de características biosociodemográficas $(n=58)$

\begin{tabular}{ccccc}
\hline & \multicolumn{5}{c}{ Nivel de estrés } \\
\hline Sexo & Total & Leve & Moderado & Profundo \\
Mujer & $47(81 \%)$ & - & - & - \\
Hombre & $11(19 \%)$ & - & - & - \\
Situación laboral & & & - & - \\
Trabaja & $14(24 \%)$ & - & - & - \\
No trabaja & $44(76 \%)$ & - & - & - \\
Estilos de vida & $\bar{x} 6$ hrs. $\pm 1,1$ & - & - & - \\
Horas de sueño & $\bar{x} 2,8$ hrs. $\pm 1,8$ & - & - & - \\
Horas de ocio & $\bar{x} 2$ hrs. $\pm 2,6$ & - & - & \\
Ejercicio & 29 & 0 & 21 & 8 \\
$\mathrm{~N}^{0}$ de sesiones Midfullnes & 2 & 1 & 1 & 0 \\
0 & 4 & 0 & 4 & 0 \\
1 & 13 & 1 & 11 & 1 \\
2 & 10 & 1 & 9 & 0 \\
\hline 3 & & &
\end{tabular}

Fuente: elaboración propia a partir de los datos.

\section{Pruebas de normalidad}

Las pruebas de normalidad a las variables de la escala SISCO son realizadas con el fin de identificar si es posible efectuar un análisis paramétrico o se debe recurrir a análisis no paramétricos. Para lo anterior, fue implementada la prueba de ShapiroWilks (tabla 2). Solo son sometidas a contraste las variables escalares, es decir, las dimensiones de la escala antes mencionada. La cantidad de sesiones a las que se asistieron los estudiantes, es una variable de tipo ordinal por lo que no se evaluará su normalidad.

En todas las dimensiones, no existen diferencias significativas con una distribución normal. Por lo tanto, fueron utilizadas pruebas paramétricas para la comparación de medias.

Tabla 2: Prueba de normalidad

\begin{tabular}{lcc}
\hline & Estadístico & $\mathrm{p}$ - valor \\
\hline Situaciones estresantes & .970 & .158 \\
Reacciones físicas & .974 & .259 \\
Reacciones psicológicas & .960 & .055 \\
Reacciones comportamentales & .974 & .247 \\
Estrategias de afrontamiento & .982 & .539 \\
\hline
\end{tabular}

Fuente: elaboración propia a partir de los datos. 


\section{Pruebas de diferencia de medias entre cantidad de sesiones asistidas}

Para comparar las medias, según la cantidad de sesiones a las que asistieron los estudiantes (dada la naturaleza ordinal de la variable en cuestión) fue realizada la prueba ANOVA de un factor. En primer lugar, se presenta la prueba de homogeneidad de varianzas para evaluar el supuesto de homocedasticidad (igualdad de varianzas) entre los grupos. En este análisis, la hipótesis nula (Ho) propone que: Todos los grupos tienen el mismo nivel de estrés. La hipótesis alternativa (Ha) plantea que: Al menos uno de los grupos tiene un nivel de estrés diferente de los demás. En la tabla 3 , se aprecia que en ninguna de las dimensiones afectó el supuesto de igualdad de varianzas $(p>.05)$, por lo que fue analizada la prueba de Tukey para comparar pares de grupos específicos, en aquellos donde exista alguna diferencia significativa.

Tabla 3. Prueba de homogeneidad de varianzas

\begin{tabular}{ccc}
\hline & Estadístico de Levene & p-valor \\
\hline Situaciones estresantes & 1.14 & .35 \\
Reacciones físicas & .36 & .84 \\
Reacciones psicológicas & 2.29 & .07 \\
Reacciones comportamentales & .32 & .86 \\
Estrategias de afrontamiento & 0.40 & .81 \\
\hline
\end{tabular}

Fuente: elaboración propia a partir de los datos.

Conocido esto, es aplicado el ANOVA (resultados están evidenciados en la tabla 4) dejando de manifiesto que existe al menos un grupo distinto de los demás en Situaciones estresantes $(F=3.323, p=.02)$, Reacciones físicas $(F=3.76, p=.01)$ y reacciones comportamentales $(\mathrm{F}=4.28, p=.00)$.

El siguiente paso es identificar dónde están las diferencias significativas, comparando cada par de grupos según las variables donde existe al menos una diferencia significativa.

Tabla 4. Prueba de diferencia de medias para dimensiones de SISCO según sesiones asistidas

\begin{tabular}{cccccccc}
\hline & \multicolumn{9}{c}{ Cantidad de veces asistidas } & \multirow{2}{*}{ F } & p \\
\cline { 2 - 6 } & 0 & 1 & 2 & 3 & 4 & & \\
\hline Situaciones estresantes & 3.60 & 2.88 & 3.19 & 3.08 & 3.09 & 3.23 & .02 \\
Reacciones físicas & 3.80 & 2.75 & 3.33 & 3.17 & 2.89 & 3.76 & .01 \\
Reacciones psicológicas & 3.39 & 3.20 & 3.40 & 2.83 & 2.54 & 2.53 & .05 \\
Reacciones comportamentales & 3.09 & 2.88 & 3.00 & 2.65 & 2.02 & 4.28 & .00 \\
Estrategias de afrontamiento & 3.26 & 2.67 & 3.08 & 2.95 & 3.08 & 0.79 & .53 \\
\hline Fuente: elaboración propia a partir de los datos. & & & & & &
\end{tabular}

Dado que las varianzas son iguales (Tabla 5) es utilizada la prueba de Tukey para estas comparaciones.

En el caso de las situaciones estresantes, llama la atención que no hay pares de variables con diferencias significativas. Sin embargo, quienes no asistieron a ninguna sesión tienen una media superior a quienes fueron al menos a 1. 
En el caso de las variables reacciones físicas y comportamentales, existen diferencias entre quienes asistieron a 0 sesiones con quienes asistieron a 4 . Si bien este proyecto está inserto en pregrado y en actividades de simulación, es posible proyectar el efecto positivo que puede tener la futura vida laboral de estos estudiantes, puesto que en situaciones reales los comportamientos inadecuados o erráticos puede desencadenar errores lamentables al estar a cargo de personas.

De este modo, podemos concluir que en general en estas variables las diferencias principales son entre quienes asistieron a 4 sesiones con quienes no asistieron a ninguna. No se encontró diferencias (por ejemplo) entre quienes asistieron a 0 sesiones y quienes estuvieron en 1, 2 o 3 . Por lo mismo, es importante recalcar que esta sería la cantidad de sesiones necesaria para generar un cambio en el nivel de estrés de los participantes.

Tabla 5. Pruebas de comparaciones post hoc

\begin{tabular}{|c|c|c|c|c|}
\hline Dimensión & $\mathrm{I}$ & $\mathrm{J}$ & Dif. de medias (I-J) & p-valor \\
\hline \multirow[t]{10}{*}{ Situaciones estresantes } & 0 & 1 & .73 & .40 \\
\hline & & 2 & .42 & .64 \\
\hline & & 3 & .53 & .05 \\
\hline & & 4 & .52 & .10 \\
\hline & 1 & 2 & -.31 & .97 \\
\hline & & 3 & -.20 & .99 \\
\hline & & 4 & -.21 & .99 \\
\hline & 2 & 3 & .11 & .99 \\
\hline & & 4 & .10 & .99 \\
\hline & 3 & 4 & -.01 & .99 \\
\hline \multirow[t]{10}{*}{ Reacciones físicas } & 0 & 1 & 1.05 & .34 \\
\hline & & 2 & .47 & .78 \\
\hline & & 3 & .63 & .11 \\
\hline & & 4 & $.91^{*}$ & .02 \\
\hline & 1 & 2 & -.58 & .90 \\
\hline & & 3 & -.42 & .95 \\
\hline & & 4 & -.14 & .99 \\
\hline & 2 & 3 & .17 & .99 \\
\hline & & 4 & .44 & .86 \\
\hline & 3 & 4 & .28 & .91 \\
\hline \multirow[t]{7}{*}{ Reacciones comportamentales } & 0 & 1 & .21 & .99 \\
\hline & & 2 & .09 & .99 \\
\hline & & 3 & .43 & .39 \\
\hline & & 4 & $1.07^{*}$ & .00 \\
\hline & 1 & 2 & -.13 & .99 \\
\hline & & 3 & .22 & .99 \\
\hline & & 4 & .86 & .55 \\
\hline
\end{tabular}




\begin{tabular}{rrrrr}
\hline 2 & 3 & .35 & .92 \\
& & 4 & .98 & .16 \\
\cline { 2 - 5 } & 3 & 4 & .64 & .24 \\
\hline
\end{tabular}

Fuente: elaboración propia a partir de los datos.

\section{Pruebas de diferencia de medias para otras variables}

Realización de pruebas T para muestras independientes con el objetivo de identificar dimensiones en la percepción de estrés según distintas variables demográficas. Sólo fueron encontradas diferencias para el género de las personas (Tabla 6).

Existen diferencias significativas entre géneros en situaciones estresantes $(p=0.02)$, Reacciones físicas $(p=0.03)$ y Estrategias de afrontamiento $(p=0.01)$. En los tres casos, son las mujeres quienes tienen las medias más altas. De este modo, son ellas quienes perciben mayores situaciones estresantes, tienen mayores reacciones físicas, pero poseen mayores estrategias para afrontarlas.

Tabla 6. Prueba de diferencia de medias para dimensiones de SISCO por género

\begin{tabular}{|c|c|c|c|c|}
\hline & \multicolumn{2}{|c|}{ Medias } & \multirow{2}{*}{$\mathrm{t}$} & \multirow{2}{*}{ p-valor } \\
\hline & Mujer & Hombre & & \\
\hline Situaciones estresantes & 3.43 & 2.95 & 2.47 & .02 \\
\hline Reacciones físicas & 3.55 & 2.94 & 2.26 & .03 \\
\hline Reacciones psicológicas & 3.19 & 2.78 & 1.42 & .16 \\
\hline Reacciones comportamentales & 2.80 & 2.77 & .09 & .93 \\
\hline Estrategias de afrontamiento & 3.24 & 2.65 & 2.82 & .01 \\
\hline
\end{tabular}

Fuente: elaboración propia a partir de los datos.

\section{DISCUSIÓN}

En Latinoamérica el estrés académico en la categoría moderada posee un porcentaje del $67 \%$ ee los estudiantes de las muestras utilizadas en diferentes estudios (2). En Chile, un de los estudios más recientes (realizado en la Universidad de los Lagos, sur del país, año 2015), evidencia que el $98 \%$ de sus estudiantes está afectado por el estrés, en las carreras del área de la salud (1). Según señalan algunas investigaciones las causas del estrés son las siguientes: situaciones en las que son evaluados (exámenes e intervenciones en público) y excesiva demanda de trabajo y deficiencias metodológicas $(1,2,13,14)$. En relación a los resultados obtenidos, el $79 \%$ de los estudiantes evaluados en la carrera de enfermería presentan un nivel de estrés moderado.

Para encontrar posibles respuestas a la problemática, López desarrolla un estudio donde el principal resultado es la disminución del agotamiento en sus participantes luego de 9 sesiones de Mindfulness. Con lo anterior, se puede relacionar la investigación del autor con los antecedentes que presenta la literatura crítica, utilizando como base el programa MBSR (desarrollo de 8 sesiones para el logro de un efecto significativo en el nivel de estrés) ${ }^{(15)}$. En la presente investigación, el logro de los efectos en los estudiantes al participar en 4 sesiones (parte de las dimensiones 
medidas en la encuesta SISCO), no son significativas para todos los aspectos medidos.

Lo mismo ocurre en el estudio de los autores Galante et al, quienes realizaron 8 semanas de intervención ${ }^{(16)}$. Según la evidencia obtenida por Harvard Business School "[..] el Mindfulness lograría en la persona estar presente y consciente, momento a momento, con independencia de las circunstancias" (17), siendo necesarias alrededor de 8 sesiones para el logro del desarrollo de herramientas para enfrentar el estrés.

Por otra parte, el estudio realizado por Asuero et al, año 2013, concluye que la práctica del mindfulness promueve conductas de autocuidado en el $94 \%$ de las personas que lo practican ${ }^{(18)}$, asimilándose a los estudios anteriormente mencionados y en forma parcial a lo encontrado en este estudio, presentando una disminución significativa del estrés en las dimensiones reacciones físicas y comportamentales.

Los logros obtenidos en el estudio realizado por Amutio et al, a 43 estudiantes de primer y segundo curso de bachillerato, el año $2015^{(19)}$, demuestran en el grupo experimental una disminución del $24 \%$ del estrés percibido en estos estudiantes, coincidiendo con la presente investigación, donde es aplicada la escala de medición de nivel de estrés que demuestra de manera objetiva el efecto de estas técnicas sobre el estrés.

El año 2013, Regehr et al (20), en el metaanálisis concluye que las intervenciones con atención plena son eficaces para reducir el estrés en estudiantes universitarios.

Finalmente, considerando el punto de vista sociodemográfico, el estudio realizado a 101 estudiantes por Abarca el año 2016, evidencia que el sexo femenino presenta mayor afección de estrés en los primeros cursos, decreciendo a medida que avanzan en la malla curricular. Siendo coincidente con los resultados obtenidos en la investigación ${ }^{(21)}$.

Las limitaciones que presenta el estudio son las siguientes:

- La seleccción de la muestra de manera no probabilística y el bajo número de participantes, lo que no permite la generalización de los resultados.

- El tiempo de la intervención. Los talleres de técnicas de Mindfulness debían estar acotados a 4 semanas de duración, lo que impide respetar los tiempos de aprendizajes que cada estudiante necesita según sus características.

- La metodología exclusivamente cuantitativa, limita el abanico de conclusiones y aportes que pueda implicar en el área de educación.

- Medición post- intervención. Para un mayor análisis de los efectos de las intervenciones una pre y post- intervención entregaría una mayor precisión.

\section{REFERENCIAS}

1. Jerez Mendoza, M., Oyarzo Barría, C. Estrés académico en estudiantes del Departamento de Salud de la Universidad de Los Lagos Osorno. Rev. Chilena de 
neuropsiquiatría. [Internet]. 2015 Sep. [citado 2019 Feb 21]; 53 (3): 149-157. Disponible en: https://scielo.conicyt.cl/scielo.php?script=sci arttext\&pid=S0717$\underline{92272015000300002}$

2. Román Callazo C., Ortiz Rodríguez F., Hernández Rodríguez Y. Rev. Iberoamericana de educación. [Internet]. julio de 2008. [citado 2019 Febrero 07]; 46(7): 1-8 Disponible en: https://rieoei.org/historico/deloslectores/2371Collazo.pdf

3. Mejía Ortiz G., Manrique N. El estrés y su relación con las condiciones de trabajo del personal de enfermería. Investigación y Educación en enfermería. Rev. Investigación y educación en enfermería. . [Internet]. Sep. 1991 [citado 2019 Feb 21]; 9(2): 83-99. Disponible en: https://aprendeenlinea.udea.edu.co/revistas/index.php/iee/article/view/21131

4. Duarte M., Varela Moreno I., Braschi Diaferia L., Sánchez Muñoz E. Estrés en estudiantes de enfermería. Rev. Educ. Media superior [Internet]. Sep. 2017 [citado 2019 Marzo 03]; 31(2): 110-123. Disponible en: http://scielo.sld.cu/scielo.php?script=sci arttext\&pid=S0864-214120170003000134. _5. Juguera Rodríguez L., Díaz Agea J., Pérez Lapuente M., Leal Costa C., Rojo Rojo A., Echevarría Pérez P. La simulación clínica como herramienta pedagógica. Percepción de los alumnos de Grado en Enfermería en la UCAM._Rev. Enfermería global._[Internet]. Enero 2014. [citado 2019 Febrero 07]; (33): 175-190. Disponible en: http://scielo.isciii.es/pdf/eg/v13n33/docencia3.pdf

6. López Sánchez M., Ramos López L., Pato López O., López Álvarez S. La simulación clínica como herramienta de aprendizaje. [Internet]. Enero 2013. [citado 2019 Febrero 07]; 18(1): 25-29. Disponible en: Recuperado de http://www.asecma.org/Documentos/Articulos/05 181 FC Lo\%C2\%A6\%C3\%BCpez .pdf

7. Sansó N., Galiana L., Oliver A., Cuesta P., Sánchez P., Benito E. Evaluación de una intervención Mindfulness en equipos de cuidados paliativos._[Internet]. 2018. [citado 2019 Febrero 07]; 27(2): 81-88. Disponible en: https://journals.copmadrid.org/pi/art/pi2018a7

8. Gutierrez Gómez N., Becerra Cabrera J., Matinez Camacho M., García Gutierrez M. Efectos de la meditación sobre el estrés académico en estudiantes de licenciatura en fisioterapia. Rev._European Scientific Journal._[nternet]. Julio 2017. [citado 2019 Febrero 07]; 13(19): 97-107. Disponible en: https://eujournal.org/index.php/esj/article/view/9624

9. Warnecke E, Quinn S, Ogden K, Towle N, Nelson MR. A randomised controlled trial of the effects of mindfulness practice on medical student stress levels. Med Educ. [Internet]. 2011. [citado 2019 Febrero 07]; 45(4):381-388. Disponible en: https://pubmed.ncbi.nlm.nih.gov/21401686/

10. Gutiérrez N., Becerra J., Martínez M., García M._Efectos De La Meditación Sobre El Estrés Académico En Estudiantes De Licenciatura En Fisioterapia. [Internet]. 2017. [citado 2019 Febrero 07]. 13 (19) 97-107. Disponible en: http://eujournal.org/index.php/esj/article/view/9624

11. Moreno Coutiño A. Terapias Cognitivo-Conductuales de Tercera generación (TTG): La Atención plena/Mindfulness. Rev. internacional de Psicología. [Internet]. 
Julio 2012. [citado 2019 Febrero 17]; 12(1): 1-18. Disponible en: http://www.revistapsicologia.org/index.php/revista/article/view/66/63

12. Kilpatrick, L., Suyenobu, B., Smith, S., Bueller, J., Goodman, T., Creswell, J., Tillisch, K., Mayer, E., Naliboff, B. Impacto $f$ mindfulness-based stress reduction training on intrinsic brain connectivity. Rev. Neuroimagen. [Internet]. Mayo 2011. [citado 2019 Febrero 17]; 56(1): 290-298. Disponible en: https://www.ncbi.nlm.nih.gov/pmc/articles/PMC3072791/

13. Holzel, B., Carmody, J., Vangel, M., Congleton, C., Yerramsetti, S., Gard, T., Lazar, S. Stess reduction correlates with structural changes in the amygdala. Rev.Social Cognitive and affective neuroscience. [Internet]. Marzo 2010. [citado 2019 Febrero 17]; 5(1): 7-11. Disponible https://www.ncbi.nlm.nih.gov/pubmed/19776221

14. Vizoso Gómez C. Arias Gundín O._Estresores académicos percibidos por estudiantes universitarios y su relación con el burnout y el rendimiento académicos. [Internet].Junio 2016. [citado 2019 Febrero 24]; 46(2) Disponible en: https://www.researchgate.net/publication/308870326 Estresores academicos percibi dos por estudiantes universitarios y su relacion con el burnout y el rendimiento academicos

15. López Hernadéz L. Técnicas mindfulness en centros educativos. desarrollo académico y personal de sus participantes revista española de orientación y psicopedagogía. Rev. [Internet]. Enero- abril 2016. [citado 2019 Febrero 24]; 27(1): 134-146 Disponible en: disponible en: https://www.redalyc.org/pdf/3382/338246652009.pdf

16. Galante J., Dufour G., Vainre M., Wagner A.P., Stochl J., Benton A., Lathia N., Howarth E., \& Jones, P. A mindfulness-based intervention to increase resilience to stress in university students. Rev. The lance public health. [Internet] 2018 [citado 2019 Febrero 24] 3: 72-81. Disponible en: 10.1016/S2468-2667(17)30231-1

17. Harvard Business School Publishing corporation. Agilidad emocional. En: Rodriguez A., Reverté R. Inteligencia emocional midfulness. España. Editorial Reverté. 2018. p. 50-67.

18. Asuero A. Rodríguez Ribera T. Berenguera A. Moix Queraltó J. Evaluación de la efectividad de un programa de mindfulness en profesionales de atención primaria. Rev. Gaceta Sanitaria. [Internet]. Nov. - Dic. 2013 [citado 2019 Febrero 27]. 27 (6):521-528. Disponible en: https://www.sciencedirect.com/science/article/pii/S0213911113000861

19. Amutio Kareaga A., Franco Justo C., Gázquez Linares J., Mañas Mañas I. Aprendizaje y práctica de la conciencia plena en estudiantes de bachillerato para potenciar la relajación y la autoeficacia en el rendimiento escolar. Rev. Universitas Psychologica. [Internet]. Abril- Junio 20152018 [citado abril 2019]. 14(2):433-443. Disponible en: https://www.redalyc.org/pdf/647/64747729003.pdf

20. Regehr C, Glancy D, Pitts A. Interventions to reduce stress in university students: a review and meta-analysis. J Affect Disord. [Internet]. 2013. [citado abril 2019]. 148(1):1-11. Disponible en: 10.1016/j.jad.2012.11.026 
21. Abarca Ahijado C. Estudio piloto: Evaluación del estrés académico en la Facultad de Fisioterapia de Soria. [tesis Internet]. Soria: Universidad de Valladolid. Julio 2016 [citado septiembre 2019]. Disponible en: http://uvadoc.uva.es/handle/10324/20549 